\title{
Fluctuation-enhanced and conductometric gas sensing with nanocrystalline NiO thin films: A comparison
}

\author{
Umut Cindemir ${ }^{*}$, Maciej Trawka ${ }^{+}$, Janusz Smulko ${ }^{+}$, Claes-Göran Granqvist ${ }^{*}$, Lars Österlund, \\ Gunnar A. Niklasson ${ }^{*}$ \\ *Department of Engineering Sciences, Uppsala University, Uppsala, Sweden, \\ umut.cindemir@angstrom.uu.se
}

${ }^{+}$Faculty of Electronics, Telecommunications and Informatics, Gdańsk University of Technology, Gdańsk, Poland

\begin{abstract}
Nanocrystalline thin films of $\mathrm{NiO}$ were prepared by advanced reactive gas deposition, and their responses to formaldehyde, ethanol and methane gases were studied via fluctuation-enhanced and conductometric methods. Thin films with thicknesses in the 200-1700-nm range were investigated in as-deposited form and after annealing at 400 and $500{ }^{\circ} \mathrm{C}$. Morphological and structural analyses showed porous deposits with $\mathrm{NiO}$ nanocrystals having face-centered cubic structure. Quantitative changes in frequency-dependent resistance fluctuations as well as in DC resistance were recorded upon exposure to formaldehyde, ethanol and methane at $200{ }^{\circ} \mathrm{C}$. The response to formaldehyde was higher than that to ethanol while the response to methane was low, which indicates that the NiO films exhibit significant selectivity towards different gaseous species. These results can be reconciled with the fact that formaldehyde has a nucleophilic group, ethanol is an electron scavenger, and methane is hard to either reduce or oxidize. The gas-induced variations in DC resistance and resistance fluctuations were in most cases similar and consistent.
\end{abstract}




\section{Introduction}

Sensors for monitoring indoor air quality attract increasing interest, which is not surprising since people in developed countries spend up to $90 \%$ of their time inside buildings or vehicles [1]. Poor air quality is a well-known cause for a condition known as the sick building syndrome (SBS), which refers to a set of diffuse and irritative symptoms connected with the time spent indoors but without any clear relationship to any specific illness [2,3]. Poor indoor air quality can have various causes such as biological contaminants, excess of carbon dioxide, etc. [3]. Furthermore, volatile organic compounds (VOCs) are one of the major reasons for poor indoor air quality and appear to be strongly associated with the SBS and related adverse health effects. Thus the detection of VOCs is essential for maintaining healthy conditions both in homes and at workplaces. Among VOCs, formaldehyde stands out as a common chemical which is emitted from wood products, cleaning agents and pesticides [4]. There is also a correlation between formaldehyde exposure and asthma symptoms [5-7].

Design and fabrication of gas sensors based on metal oxide thin films is gaining widespread interest as a consequence of their semiconducting properties and low cost, and a large number of studies have been carried out for sensors based on, for example, $\mathrm{CuO}, \mathrm{SnO}_{2}, \mathrm{ZnO}$ and $\mathrm{WO}_{3}$ exposed to various gases [8-11]. These sensors' operating principle relies on resistance changes in the presence of gaseous species which induce surface reactions in grain boundaries and act as a donors or acceptors of electrons [8,12]. Different gaseous species can be monitored with a single sensor if its operating temperature is altered, but issues concerning poor selectivity are prevalent $[13,14]$.

Many different techniques, from wet chemical processing to physical deposition, have been used to produce nanoparticles and thin films for metal-oxide-based gas sensors [15-18]. Important parameters for such devices are related to efficient use of nanoscale properties such as structure, particle and grain size, porosity and film thickness. Advanced gas deposition (AGD) stands out as a particularly suitable method to achieve reproducible properties in samples with high porosity and surface area [19,20], since nanoparticle growth is separated from thin-film formation.

Among semiconducting metal oxides, nickel oxide (NiO) has attracted considerable attention. It is a $p$ type semiconductor with reported bandgap energy between 3.6 and $4.0 \mathrm{eV}$, and it can exhibit large changes of its conductivity owing to surface-chemical reactions [16,21-23]. Diverse manufacturing methods have been applied to produce $\mathrm{NiO}$ thin films and nanostructures, including chemical methods, sputtering and evaporation [15,24], and $\mathrm{NiO}$ films have been used in sensors for formaldehyde $\left(\mathrm{CH}_{2} \mathrm{O}\right)$ [2,21], ethanol $\left(\mathrm{C}_{2} \mathrm{H}_{5} \mathrm{OH}\right)$ [16], $\mathrm{H}_{2} \mathrm{~S}$ [15], $\mathrm{NO}_{2}$ [15], $\mathrm{NH}_{3}$ [24], $\mathrm{CO}$ [25] and $\mathrm{H}_{2}$ [26-28]. These sensors generally operate at elevated temperature to activate surface-chemical reactions $[8,15]$. The usual method to detect a gas relies on electrical properties, but modifications in optical properties have been studied too $[29,30]$.

Several principles can be utilized for metal-oxide-based gas sensing. A conventional one records changes in the DC resistance upon gas exposure and is referred to as "conductometric" sensing. This technique is popular and comparatively simple but has an inherent limitation in its recording of a single parameter. Another technique, which avoids this limitation, is based on amplification, measurement and analysis of the sensor signal's stochastic component that arises from resistance 
fluctuations during surface reactions [31-34], and which can boost both sensistivity and selectivity of gas detection [35]. The latter operation mode is called "fluctuation-enhanced sensing" (FES) [36,37] and can be implemented in two ways: in the regular one [36] the sensor is heated continuously and measurements are carried out at a stable operating temperature, whereas in another approach —referred to as the "sample-and-hold-method" [38,39]—heating is turned off and the sensor is cooled to room temperature at which the measurements are performed.

In the present work, we recorded and compared gas sensing based on DC resistance and resistance fluctuations, using the regular mode for FES, for AGD-prepared $\mathrm{NiO}$ thin-film deposits exposed to formaldehyde, ethanol and methane.

\section{Experimental techniques and procedures}

\subsection{Sample preparation}

Thin-film NiO deposits were prepared on $4 \times 25 \mathrm{~mm}^{2} \mathrm{Al}_{2} \mathrm{O}_{3}$ ceramic substrates devised for sensor applications, as shown in Fig. 1, and also on glass and Si plates for materials characterization. The $\mathrm{Al}_{2} \mathrm{O}_{3}$ substrates had preprinted Pt-Ag alloy electrodes on both sides. The upper side had two combstructured electrodes with five 2-mm-long fingers separated by $0.3-\mathrm{mm}$-wide gaps, and the reverse side had a 0.4-mm-wide heating resistor for controlling the sensor's temperature during its use. The operating temperature was measured with a platinum resistor (Pt100) glued to the substrate via silver epoxy.

$\mathrm{NiO}$ nanoparticle films were deposited by using an advanced reactive gas deposition unit (ULVAC Ltd., Japan). This unit has two chambers, one for evaporation and the other for film formation, connected via a transfer pipe. The deposition method was as follows: Ni pellets (purity 99.95\%) were placed inside a carbon crucible surrounded by an RF heating coil in the evaporation chamber, and a mixture of $\mathrm{He}$ (flow rate $20 \mathrm{ml} / \mathrm{min}$, purity 99.997\%) and $\mathrm{O}_{2}$ (flow rate $10 \mathrm{ml} / \mathrm{min}$, purity $99.998 \%$ ) was let into the evaporation chamber so that it formed a laminar flow surrounding the crucible. The induction coil was powered at $3 \mathrm{~kW}$. $\mathrm{Ni}$ atoms evaporated from the material in the crucible, subsequently formed oxidized nuclei in the $\mathrm{He}+\mathrm{O}_{2}$ mixture, and these nuclei grew into nanoparticles with [20] or without [40,41] coagulation. NiO nanoparticles were then transported into the film formation chamber through the transfer pipe as a result of a pressure difference between the two chambers. Table I shows actual values of the pressures $p_{e}$ and $p_{f}$ in the evaporation and film formation chambers, respectively. The substrates were positioned on an $\mathrm{X}-\mathrm{Y}$ stage and were moved in front of a nozzle at the upper aperture of the transfer pipe so that deposits with thicknesses given in Table I were formed; the thickest deposit was prepared as two superimposed layers. A schematic drawing of the AGD system is shown in Fig. 2, and more detailed information can be found in previous publications $[42,43]$.

Three $\mathrm{Al}_{2} \mathrm{O}_{3}$ sensor substrates were coated with $\mathrm{NiO}$ nanoparticles simultaneously and are jointly referred to as a "set". Table I shows the evaporation conditions and film thicknesses for all sets of samples. One of the members of this set was left in as-deposited state, a second member was heat treated for $12 \mathrm{~h}$ at $400{ }^{\circ} \mathrm{C}$, and the third member was heat treated for $12 \mathrm{~h}$ at $500{ }^{\circ} \mathrm{C}$. For annealing at $400{ }^{\circ} \mathrm{C}$, the temperature was ramped up from 25 to $400{ }^{\circ} \mathrm{C}$ at $\sim 3{ }^{\circ} \mathrm{C} / \mathrm{min}$ and was ramped back at $\sim 0.5$ ${ }^{\circ} \mathrm{C} / \mathrm{min}$; for annealing at $500{ }^{\circ} \mathrm{C}$, the temperature was ramped up from 25 to $500{ }^{\circ} \mathrm{C}$ at $\sim 4{ }^{\circ} \mathrm{C} / \mathrm{min}$ and was ramped back at $\sim 0.7^{\circ} \mathrm{C} / \mathrm{min}$. Furthermore, depositions were made in parallel onto glass and $\mathrm{Si}$ substrates for characterization purposes.

\subsection{Film characterization techniques}


Film thicknesses were recorded by using a Veeco Dektak 150 surface profilometer. Atomic structures were obtained by grazing-incidence x-ray diffraction (XRD) employing a Siemens D5000 diffractometer operating with $\mathrm{CuK}_{\alpha 1}$ radiation $(0.15406 \mathrm{~nm})$ in a $2 \theta$-range from 2 to $90^{\circ}$. Top-view surface microstructures as well as cross-sections of the deposits were acquired by scanning electron microscopy (SEM) using a Zeiss LEO 1550 FEG instrument, equipped with an in-lens detector, at an acceleration voltage of $10 \mathrm{kV}$.

\subsection{Gas sensing techniques}

Resistance noise was recorded for the $\mathrm{NiO}$ samples upon their exposure to formaldehyde, ethanol and methane. The measurements were carried out in a one-liter metal chamber equipped with mass-flow controllers at a gas flow that was low enough to avoid turbulence, specifically at $100 \mathrm{ml} / \mathrm{min}$. The sensors were heated during the measurements, and a stable operating temperature was then kept at 200 ${ }^{\circ} \mathrm{C}$. The sensors were investigated for each gaseous species at different concentrations in a carrier gas of synthetic air (SA; $20 \% \mathrm{O}_{2}, 80 \% \mathrm{~N}_{2}$ ); formaldehyde was tested at $0.5,1,2,5$ and $10 \mathrm{ppm}$, ethanol at 25 and $50 \mathrm{ppm}$, and methane at $50 \mathrm{ppm}$. The sole exception to this scheme was that sensors from sample set 1 were tested in ethanol only at $50 \mathrm{ppm}$.

In the beginning of each measurement, sensors were heated to $200{ }^{\circ} \mathrm{C}$ during $1 \mathrm{~h}$ in SA, and the sensors remained at this temperature in SA for another half hour. Power spectral densities (PSDs) were then recorded, as schematically illustrated in Fig. 3(a), at the end of each subsequent 30-minuteperiod. PSD spectra were obtained as averages over 100 individual recordings, each taken during a period of four seconds. The frequency ranges for the measurements were $2-202 \mathrm{~Hz}$ and $10-1610 \mathrm{~Hz}$ so that three decades could be covered for the PSDs. After recording the noise spectra, the concentration of each gas was increased to the next larger value. Values of DC resistance were recorded in conjunction with the noise measurements.

The sensor under study was put in a negative feed-back loop of a low-noise operational amplifier (MAX4478, Maxim Integrated) and was driven by a DC current as shown in Fig. 3(b). The input resistor $R$ was used to bias the sensor at DC currents of 2.65, 12.4, 56.75 and $124.5 \mu \mathrm{A}$; different values were required since the sensor's resistance was dependent on its gaseous ambience. A spectrum analyzer (Stanford SR760) was used to record PSDs of the voltage fluctuations; these fluctuations were proportional to the sensor's resistance fluctuations. During the FES experiments, the background noise of the system was negligible compared with the noise of the sensor.

The sensor's $D C$ resistance response $G_{d c}$ is independent of voltage bias and is defined as [42]

$$
G_{d c}=\frac{R_{t g}}{R_{s a}}
$$

where $R_{\mathrm{tg}}$ denotes the resistance after exposure to the specific "target" gas for 30 minutes and $R_{\mathrm{s} a}$ is the resistance during exposure to SA only. The normalized noise response $G_{n}$ is defined according to [42]

$$
G_{n}(f)=\frac{\left(s_{u}(f) / U_{D C}^{2}\right)_{t g}}{\left(s_{u}(f) / U_{D C}^{2}\right)_{s a}},
$$

where $S_{u}(f) / U^{2}{ }_{D C}$ denotes the power spectral density normalized by the squared DC voltage, $U^{2}{ }_{D C}$, across the sensor, and $f$ is frequency. The normalization by $U_{D C}^{2}$ was performed in order to remove a dependence on bias conditions. It was verified by experiments that the noise spectrum scaled with the squared DC voltage. 


\section{Results}

\subsection{Film characterization data}

Figure 4 shows an example of SEM images, specifically a top view (panel a) and a cross-section (panel b) of a NiO film prepared by AGD. A porous morphology is apparent, which is an attractive feature for gas sensing. Film thickness determination by SEM and surface profilometry were found to be in good agreement.

XRD data in Fig. 5 indicate that the NiO film has a cubic NiO structure corresponding to reference pattern ICDD:04-001-9373 (International Centre for Diffraction Data) with a lattice parameter of $0.417 \mathrm{~nm}$. An average crystallite diameter $D$ was inferred from the <200> peaks by use of Scherrer's formula. Specifically, we found $D$ to be $3.7 \mathrm{~nm}$ for the as-deposited film and 5.9 and $11 \mathrm{~nm}$ after annealing at 400 and $500{ }^{\circ} \mathrm{C}$, respectively, i.e., $D$ grew markedly as the sample was heat treated at increasing temperature.

DC resistance was measured for specimens in each of the three sample sets after heating them in SA. As shown in Fig. 6, the resistance grows for increasing annealing temperature with the change being particularly strong after heat treatment at $500{ }^{\circ} \mathrm{C}$.

\subsection{Gas sensing data based on resistance fluctuations}

Normalized noise responses were recorded as described above and data are shown in Fig. 7(a) for ethanol and methane and in Fig. 7(b) for formaldehyde. The data display an approximate 1/f-behavior, especially at intermediate frequencies. Clearly the response to methane is practically nil despite the concentration of this gas being as large as $50 \mathrm{ppm}$. Ethanol, on the other hand, displays a clear response in spite of the concentration being half as large. The results for formaldehyde are particularly interesting, and the response is very clear despite the fact that the gas concentration was as low as 1 ppm. It is thus evident that the NiO films are highly selective to gaseous species.

Data on $G_{n}$ were evaluated from the normalized PSDs at $f=10 \mathrm{~Hz}$ and data are shown for formaldehyde in Fig. 8 and for ethanol and methane in Fig. 9. The response to formaldehyde is largest for the thinnest film (Fig. 8a), whereas annealing at $500{ }^{\circ} \mathrm{C}$ and associated grain growth eliminates the response (Fig. 8b). We note that noise data are expected to depend on sample preparation, and it has been reported repeatedly that there is a direct link between $1 / f$ noise and defect density [44]. Higher annealing temperature means less defects and hence a lower noise response, as also observed in fig. 8b. Furthermore, the intensity of $1 / f$ noise is inversely proportional to the number of charges involved in its generation [45] - i.e., to the sample volume. In our case this leads to the higher noise for lower film thicknesses in fig. 8a.

However, the response to ethanol appears erratic with regard to film thickness (Fig 9a), while annealing of the thinnest film also in this case yields a consistent decrease of the sensitivity. The response to methane is observed to be extremely weak.

\subsection{Gas sensing data based on DC resistance}

DC resistance was recorded along with the noise measurements, and Fig. 10 shows $G_{d c}$ for NiO films exposed to formaldehyde. Panel (a) refers to data taken for as-deposited samples of different thicknesses and shows that the thinnest film has the largest response, whereas panel (b) indicates that the response for the thinnest film is largest when it is in its as-deposited state whereas annealing and associated grain growth at successively larger temperature makes $G_{d c}$ drop. It should be noted that the stability of the resistive signal—i.e., the change of the resistance during averaging —is improved after 
annealing as apparent from the smaller error bars. Figure 11 displays corresponding data for $\mathrm{NiO}$ films exposed to ethanol and methane. Panel (a), reporting data for as-deposited films, shows that the response to ethanol is largest for the intermediate thickness while the response to methane consistently is very weak, whereas panel (b) shows that the response to ethanol goes down upon annealing of the thinnest film, which is consistent with the behavior upon exposure to formaldehyde.

\section{Discussion and conclusions}

The data presented above show that both resistance fluctuations and DC resistance can change significantly when nanocrystalline $\mathrm{NiO}$ films are exposed to different gases. The underlying mechanism for these effects is oxygen adsorption and dissociation with ensuing electron transfer reactions by oxidation of gases adsorbed on the surface of the grains in the $\mathrm{NiO}$ films. Since $\mathrm{NiO}$ is a p-type semiconductor, pre-adsorbed oxygen species capture electrons from $\mathrm{Ni}$ atoms in the film thereby increasing the number of holes and decreasing its resistance [46]. During gas sensing, the oxidation and fragmentation of gases adsorbed on the sensor's surface can be expressed, schematically, as [21]

$$
\text { gas molecule }+\mathrm{O}^{-} / \mathrm{O}_{2}^{-} / \mathrm{O}^{2-} \rightarrow \mathrm{CO}_{2}+\mathrm{H}_{2} \mathrm{O}+e^{-} .
$$

The outcome of this reaction is an increase in the number of electrons $\left(e^{-}\right)$on the surface of the $\mathrm{NiO}$ film, and hence the number of majority carriers (i.e., holes) is decreased by charge recombination with the electrons produced in the oxidation of the gas molecules; an increase in the resistance is consequently observed. In case of electron acceptors, such as ethanol, competing electron scavenging and molecular fragmentation takes place, and a weaker response is expected. The diffusion of the molecule fragments on the $\mathrm{NiO}$ films' surfaces (in particular along grain boundaries), and their effect on the resistance, is also a time-dependent stochastic process. Thus measurements of resistance noise can be used to distinguish different gaseous species. The observed 1/f-type resistance noise in general depends on the intensity of these random processes, which also are influenced by the type of ambient gas. In the present case, experiments were conducted to monitor the response of $\mathrm{NiO}$ films to formaldehyde, ethanol and methane. These gases have principally different properties with formaldehyde having a nucleophilic group, ethanol being an electron scavenger, whereas methane in comparison is inert towards oxidation-reduction reactions. These features of the mentioned molecules can explain the selectivity of the $\mathrm{NiO}$ sensors.

Voltage fluctuations across the sensor depend on its bias conditions and are proportional to the square of the DC voltage [47]. Therefore the PSD of the voltage noise was normalized with respect to the square of the DC voltage across the sensor. This leads to $S_{u}(f) /\left(U_{\mathrm{DC}}\right)^{2}$ being independent of the measurement system and equal to $S_{\mathrm{r}}(f) /(R)^{2}$, representing the normalized power spectral density of resistance fluctuations in a gas sensor having a DC resistance $R$. A more thorough discussion on various set-ups for low-frequency noise measurements in resistive sensors can be found elsewhere $[47,48]$.

The NiO sensors displayed selective gas detection both in conductometric and noise-based measurements. The response to formaldehyde was higher than to ethanol, which is consistent with dual oxidation and electron scavenging occurring for ethanol and yielding fewer electrons (reaction 3). We suggest that the reason why the $\mathrm{NiO}$ films with intermediate thickness showed the largest response towards ethanol is related to structure-sensitive surface reactions being most prominent for that film and giving the highest oxidation rate, and thereby the highest net electron concentration, thus yielding the largest change of hole conduction due to electron recombination. No significant response 
whatsoever was detected for methane. Furthermore, the two gas detection methods were compared. For most cases, the responses were similar and consistent with regard to gaseous species, film thickness and dependence on annealing temperature. We suppose that these types of responses can be different for other gases, as indicated elsewhere [32,42].

Finally, we remark that advanced stochastic signal analysis [32] may further improve the fluctuationenhanced gas sensing, and we note that the FES sensitivity experiments were limited by the utilization of a single frequency. The use of the whole spectral pattern, as well as the sampling-and-hold FES technique, has been shown to increase sensitivity and selectivity.

\section{Acknowledgements}

Financial support was received from the European Research Council under the European Community's Seventh Framework Program (FP7/2007-2013)/ERC, Grant Agreement No. 267234 (“GRINDOOR”) and from the European Union's Horizon 2020 research and innovation programme under the Marie Skłodowska-Curie grant agreement No 645758 (“TROPSENSE”).

\section{References}

[1] J.A. Leech, W.C. Nelson, R.T. Burnett, S. Aaron, M.E. Raizenne, It’s about time: a comparison of Canadian and American time-activity patterns., J. Expo. Anal. Environ. Epidemiol. 12 (2002) 427-432. doi:10.1038/sj.jea.7500244.

[2] C.-Y. Lee, C.-M. Chiang, Y.-H. Wang, R.-H. Ma, A self-heating gas sensor with integrated $\mathrm{NiO}$ thin-film for formaldehyde detection, Sensors Actuators B Chem. 122 (2007) 503-510. doi:10.1016/j.snb.2006.06.018.

[3] M. Murphy, Sick building syndrome and the problem of uncertainty: Environmental politics, technoscience, and women workers., Duke University Press, Durham, NC, 2006.

[4] K.C. Gupta, A.G. Ulsamer, P.W. Preuss, Formaldehyde in indoor air: Sources and toxicity, Environ. Int. 8 (1982) 349-358. doi:10.1016/0160-4120(82)90049-6.

[5] J.J. Quackenboss, M.D. Lebowitz, J.P. Michaud, D. Bronnimann, Formaldehyde exposure and acute health effects study, Environ. Int. 15 (1989) 169-176. doi:10.1016/0160-4120(89)900238.

[6] K.B. Rumchev, J.T. Spickett, M.K. Bulsara, M.R. Phillips, S.M. Stick, Domestic exposure to formaldehyde significantly increases the risk of asthma in young children, Eur. Respir. J. 20 (2002) 403-408. doi:10.1183/09031936.02.00245002.

[7] H. Nordman, H. Keskinen, M. Tuppurainen, Formaldehyde asthma-Rare or overlooked?, J. Allergy Clin. Immunol. 75 (1985) 91-99. doi:10.1016/0091-6749(85)90018-1.

[8] N. Barsan, D. Koziej, U. Weimar, Metal oxide-based gas sensor research: How to?, Sensors Actuators B Chem. 121 (2007) 18-35. doi:10.1016/j.snb.2006.09.047.

[9] G. Eranna, B.C. Joshi, D.P. Runthala, R.P. Gupta, Oxide Materials for Development of Integrated Gas Sensors-A Comprehensive Review, Crit. Rev. Solid State Mater. Sci. (2010). http://www.tandfonline.com/doi/abs/10.1080/10408430490888977 (accessed May 23, 2016).

[10] M. Tiemann, Porous metal oxides as gas sensors., Chemistry-A European Journal. 13 (2007) 8376-88. doi:10.1002/chem.200700927.

[11] G. Korotcenkov, The role of morphology and crystallographic structure of metal oxides in response of conductometric-type gas sensors, Mater. Sci. Eng. R Reports. 61 (2008) 1-39. doi:10.1016/j.mser.2008.02.001.

[12] N. Barsan, U. Weimar, Conduction model of metal oxide gas sensors, J. Electroceramics. 7 
(2001) 143-167. doi:10.1023/A:1014405811371.

[13] A. Heilig, N. Bârsan, U. Weimar, M. Schweizer-Berberich, J.W. Gardner, W. Göpel, Gas identification by modulating temperatures of $\mathrm{SnO}_{2}$-based thick film sensors, Sensors Actuators B Chem. 43 (1997) 45-51. doi:10.1016/S0925-4005(97)00096-8.

[14] S. Nakata, S. Akakabe, M. Nakasuji, K. Yoshikawa, Gas sensing based on a nonlinear response: Discrimination between hydrocarbons and quantification of individual components in a gas mixture, Anal. Chem. 68 (1996) 2067-2072. doi:10.1021/ac9510954.

[15] C. Luyo, R. Ionescu, L.F. Reyes, Z. Topalian, W. Estrada, E. Llobet, C.G. Granqvist, P. Heszler, Gas sensing response of $\mathrm{NiO}$ nanoparticle films made by reactive gas deposition, Sensors Actuators B Chem. 138 (2009) 14-20. doi:10.1016/j.snb.2008.11.057.

[16] Y. Du, W. Wang, X. Li, J. Zhao, J. Ma, Y. Liu, G. Lu, Preparation of NiO nanoparticles in microemulsion and its gas sensing performance, Mater. Lett. 68 (2012) 168-170. doi:10.1016/j.matlet.2011.10.039.

[17] J.A. Kemmler, S. Pokhrel, J. Birkenstock, M. Schowalter, A. Rosenauer, N. Bârsan, U. Weimar, L. Mädler, Quenched, nanocrystalline $\mathrm{In}_{4} \mathrm{Sn}_{3} \mathrm{O}_{12}$ high temperature phase for gas sensing applications, Sensors Actuators B Chem. 161 (2012) 740-747. doi:10.1016/j.snb.2011.11.026.

[18] S. Das, V. Jayaraman, $\mathrm{SnO}_{2}$ : A comprehensive review on structures and gas sensors, Prog. Mater. Sci. 66 (2014) 112-255. doi:10.1016/j.pmatsci.2014.06.003.

[19] J.L. Solis, A. Hoel, L.B. Kish, C.G. Granqvist, S. Saukko, V. Lantto, Gas-sensing properties of nanocrystalline $\mathrm{WO}_{3}$ films made by advanced reactive gas deposition, J. Am. Ceram. Soc. 84 (2004) 1504-1508. doi:10.1111/j.1151-2916.2001.tb00868.x.

[20] C.G. Granqvist, Ultrafine metal particles, J. Appl. Phys. 47 (1976) 2200. doi:10.1063/1.322870.

[21] I. Castro-Hurtado, J. Herrán, G.G. Mandayo, E. Castaño, Studies of influence of structural properties and thickness of $\mathrm{NiO}$ thin films on formaldehyde detection, Thin Solid Films. 520 (2011) 947-952. doi:10.1016/j.tsf.2011.04.180.

[22] X. Song, L. Gao, S. Mathur, Synthesis, Characterization, and gas sensing properties of porous nickel oxide nanotubes, J. Phys. Chem. C. 115 (2011) 21730-21735. doi:10.1021/jp208093s.

[23] A.M. Soleimanpour, A.H. Jayatissa, G. Sumanasekera, Surface and gas sensing properties of nanocrystalline nickel oxide thin films, Appl. Surf. Sci. 276 (2013) 291-297. doi:10.1016/j.apsusc.2013.03.085.

[24] J. Wang, P. Yang, X. Wei, Z. Zhou, Preparation of NiO two-dimensional grainy films and their high-performance gas sensors for ammonia detection, Nanoscale Res. Lett. 10 (2015) 119. doi:10.1186/s11671-015-0807-5.

[25] I. Hotovy, J. Huran, P. Siciliano, S. Capone, L. Spiess, V. Rehacek, The influences of preparation parameters on $\mathrm{NiO}$ thin film properties for gas-sensing application, Sensors Actuators B Chem. 78 (2001) 126-132. doi:10.1016/S0925-4005(01)00802-4.

[26] W. Shin, M. Matsumiya, N. Izu, N. Murayama, Hydrogen-selective thermoelectric gas sensor, Sensors Actuators B Chem. 93 (2003) 304-308. doi:10.1016/S0925-4005(03)00225-9.

[27] I. Rýger, G. Vanko, T. Lalinský, P. Kunzo, M. Vallo, I. Vávra, T. Plecenik, Pt/NiO ring gate based Schottky diode hydrogen sensors with enhanced sensitivity and thermal stability, Sensors Actuators B Chem. 202 (2014) 1-8. doi:10.1016/j.snb.2014.05.052.

[28] Z. Wang, Z. Li, J. Sun, H. Zhang, W. Wang, W. Zheng, C. Wang, Improved hydrogen monitoring properties based on $p-\mathrm{NiO} / n-\mathrm{SnO}_{2}$ heterojunction composite nanofibers, J. Phys. Chem. C. 114 (2010) 6100-6105. doi:10.1021/jp9100202.

[29] A. Martucci, N. Bassiri, M. Guglielmi, L. Armelao, S. Gross, J.C. Pivin, NiO-SiO 2 sol-gel nanocomposite films for optical gas sensor, J. Sol-Gel Sci. Technol. 26 (1-3) 993-996. 
doi:10.1023/A:1020717614995.

[30] G. Mattei, P. Mazzoldi, M.L. Post, D. Buso, M. Guglielmi, A. Martucci, Cookie-like Au/NiO nanoparticles with optical gas-sensing properties, Adv. Mater. 19 (2007) 561-564. doi:10.1002/adma.200600930.

[31] L.B. Kish, Y. Li, S. Member, J.L. Solis, W.H. Marlow, R. Vajtai, C.G. Granqvist, V. Lantto, J.M. Smulko, G. Schmera, Fluctuation-enhanced sensing with Taguchi sensors, IEEE Sens. J. 5 (2005) 671-676.

[32] C. Kwan, G. Schmera, J.M. Smulko, L.B. Kish, P. Heszler, C.G. Granqvist, Advanced agent identification with fluctuation-enhanced sensing, IEEE Sens. J. 8 (2008) 706-713.

[33] S. Gomri, J.-L. Seguin, K. Aguir, Modeling on oxygen chemisorption-induced noise in metallic oxide gas sensors, Sensors Actuators B Chem. 107 (2005) 722-729. doi:10.1016/j.snb.2004.12.003.

[34] S. Gomri, J.-L. Seguin, J. Guerin, K. Aguir, A mobility and free carriers density fluctuations based model of adsorption-desorption noise in gas sensor, J. Phys. D. Appl. Phys. 41 (2008) 065501. doi:10.1088/0022-3727/41/6/065501.

[35] R. Macku, J. Smulko, P. Koktavy, M. Trawka, P. Sedlak, Analytical fluctuation enhanced sensing by resistive gas sensors, Sensors Actuators B Chem. 213 (2015) 390-396. doi:10.1016/j.snb.2015.02.114.

[36] H.-C. Chang, L.B. Kish, M.D. King, C. Kwan, Fluctuation-enhanced sensing of bacterium odors, Sensors Actuators B Chem. 142 (2009) 429-434. doi:10.1016/j.snb.2009.04.005.

[37] L.B. Kish, R. Vajtai, C.G. Granqvist, Extracting information from noise spectra of chemical sensors: Single sensor electronic noses and tongues, Sensors Actuators B Chem. 71 (2000) 5559. doi:10.1016/S0925-4005(00)00586-4.

[38] J.L. Solis, L.B. Kish, R. Vajtai, C.G. Granqvist, J. Olsson, J. Schnürer,V. Lantto, Identifying natural and artificial odours through noise analysis with a sampling-and-hold electronic nose, Sensors Actuators B Chem. 77 (2001) 312-315. doi:10.1016/S0925-4005(01)00698-0.

[39] J. Smulko, M. Trawka, Gas selectivity enhancement by sampling-and-hold method in resistive gas sensors, Sensors Actuators B Chem. 219 (2015) 17-21. doi:10.1016/j.snb.2015.04.120.

[40] J. Söderlund, L.B. Kiss, G.A. Niklasson, C.G. Granqvist, Lognormal size distributions in particle growth processes without coagulation, Phys. Rev. Lett. 80 (1998) 2386-2388. doi:10.1103/PhysRevLett.80.2386.

[41] L.B. Kiss, J. Söderlund, G.A. Niklasson, C.G. Granqvist, New approach to the origin of lognormal size distributions of nanoparticles, Nanotechnology 10 (1999) 25-28.

[42] J. Ederth, J.M. Smulko, L.B. Kish, P. Heszler, C.G. Granqvist, Comparison of classical and fluctuation-enhanced gas sensing with $\mathrm{Pd}_{x} \mathrm{WO}_{3}$ nanoparticle films, Sensors Actuators B Chem. 113 (2006) 310-315. doi:10.1016/j.snb.2005.03.009.

[43] J. Ederth, L.B. Kish, E. Olsson, C.G. Granqvist, In situ electrical transport during isothermal annealing of nanocrystalline gold films, J. Appl. Phys. 91 (2002) 1529-1535. doi:10.1063/1.1427399.

[44] D.M. Fleetwood, N. Giordano, Direct link between $1 / f$ noise and defects in metal films, Phys. Rev. B 31 (1985) 1157-1160. doi:10.1103/PhysRevB.31.1157.

[45] F.N. Hooge, T.G.M. Kleinpenning, L.K.J. Vandamme, Rep. Prog. Phys. 44 (1981) 479-532.

[46] D. Kohl, Function and applications of gas sensors, J. Phys. D. Appl. Phys. 34 (2001) R125R149. doi:10.1088/0022-3727/34/19/201.

[47] M. Kotarski, J. Smulko, Noise measurement set-ups for fluctuations-enhanced gas sensing, Metrol. Meas. Syst. 16 (2009) 457-464.

[48] B. Ayhan, C. Kwan, J. Zhou, L.B. Kish, K.D. Benkstein, P.H. Rogers, S. Semancik, 
Fluctuation enhanced sensing (FES) with a nanostructured, semiconducting metal oxide film for gas detection and classification, Sensors Actuators B Chem. 188 (2013) 651-660. doi:10.1016/j.snb.2013.07.056. 